\title{
Osteofibrous dysplasia-like adamantinoma treated via intercalary segmental resection with partial cortex preservation using pedicled vascularized fibula graft: a case report
}

\author{
Yuji Yamamura', Makoto Emori ${ }^{1 *}$ (D), Nobuyuki Takahashi ${ }^{1}$, Mitsumasa Chiba', Junya Shimizu', Yasutaka Murahashi', \\ Shintaro Sugita ${ }^{2}$, Kousuke lba ${ }^{1}$, Tadashi Hasegawa ${ }^{2}$ and Toshihiko Yamashita ${ }^{1}$
}

\begin{abstract}
Background: Morphologically, osteofibrous dysplasia-like adamantinoma is thought to be intermediate between osteofibrous dysplasia and adamantinoma. Its treatment is not well established owing to its rarity.

Case presentation: We report about of a 10-year-old girl with osteofibrous dysplasia-like adamantinoma initially diagnosed as osteofibrous dysplasia and treated via intercalary segmental resection with partial cortex preservation using a pedicled vascularized fibula graft for reconstruction. Bone union was observed 9 weeks after surgery. Twenty-two months after the definitive surgery, no recurrence was observed.
\end{abstract}

Conclusion: This case illustrates the upgrade from osteofibrous dysplasia to osteofibrous dysplasia-like adamantinoma. The surgical method may aid the treatment of osteofibrous dysplasia-like adamantinoma with incomplete cortex involvement of the tumor.

Keywords: Osteofibrous dysplasia, Vascularized fibula graft, Bone tumor

\section{Introduction}

An osteofibrous dysplasia (OFD) is a rare, benign, selflimited fibrous osseous lesion that exclusively affects the midshaft of the tibia and mostly occurs in children [1]. Pain and swelling are the most common initial symptoms. OFD usually progresses until the patient is 10-year old with stabilization at about 15 years of age and occasionally regresses spontaneously after puberty. Some lesions may be periodically aggressive with anterior tibia bowing deformity. Although most patients require close

\footnotetext{
* Correspondence: emrmkt@yahoo.co.jp

'Department of Orthopedic Surgery, Sapporo Medical University School of Medicine, West 16, South 1, Chuo- ku, Sapporo, Hokkaido 060-8543, Japan Full list of author information is available at the end of the article
}

observation only, surgery is indicated when bone destruction, deformity, and local symptoms become severe.

OFD, OFD-like adamantinoma (AD), and classic AD are postulated to represent a spectrum of morphologically similar diseases. Histologically, OFD-like AD lies between $\mathrm{OFD}$ and $\mathrm{AD}$. It was first thought to be a precursor to $\mathrm{AD}$ [2] but is now considered a progression of OFD [3-6]. The clinical presentation of OFD-like AD is similar to that of OFD. Typically, OFD-like AD develops during the first two decades of life, with mean age of 13.4 years $[6,7]$. OFD-like AD is exclusively intracortical in the tibia with frequent synchronous involvement of the ipsilateral fibula.

The treatment of OFD-like AD is not well established owing to the scarcity of cases. It is usually based on the 
symptoms and the extent of the lesion, but broadly follows that for OFD with observation with plain radiographs at six monthly to annual intervals for a minimum of 10 years [6]. Although there is no evidence of progression to $\mathrm{AD}$, the recommended treatment for OFD-like $\mathrm{AD}$ in recent years is radical segmental resection rather than curettage, as it may prevent local recurrence $[5,6]$. The most popular reconstructive options after segmental excision of a bone tumor include allografts, vascularized fibula grafts (VFGs), combined allografts and VFGs, segmental endoprostheses, extracorporeal devitalized autografts, and segmental transport. A VFG is recommended to reconstruct tibial bone defects using only a VFG, but complications such as pseudarthrosis and fracture remain problematic [8].

We report a case of OFD-like AD initially diagnosed as OFD and treated via segmental resection with partial cortex preservation using a pedicled VFG (PVFG) and locking plate for reconstruction.

\section{Case presentation}

A 10-year-old girl presented with a 3-month history of pain in her left lower leg after bruising. She had no medical conditions and was physically active. On physical examination, she had a bony lump in the middle third of the left tibia with some tenderness. An X-ray of the left leg showed a 6 -cm mass with multiple osteolytic and sclerotic lesions in the thickened anterior diaphysis of the left tibia (Fig. 1a, b). No periosteal reaction was observed. Computed tomography revealed that the $6-\mathrm{cm}$ mass was confined to the cortex of the tibia (Fig. 1c). On magnetic resonance imaging (MRI), it was heterogeneously hypointense and isointense on a T1-weighted sagittal image and heterogeneously hyperintense on a $\mathrm{T} 2$ sagittal image; it measured $63 \mathrm{~mm}$ in the tibia with incomplete involvement of the marrow cavity (Fig. 1d, e). The mass was highly suspected to be an OFD, and open biopsy was performed.

Histologic examination of the biopsy specimen revealed that immature bone trabeculae were surrounded partly by prominent osteoblastic rimming (Fig. 2). Cytokeratin (AE1/AE3)-positive epithelial cells were sparsely distributed throughout the specimen, but clusters of epithelial tissues were absent. Based on the combined results of imaging and histologic analysis, OFD was diagnosed.

The patient was followed via observation only regardless of pain. However, she had difficulty walking owing to increasing pain 4 months after diagnosis. MRI revealed a slight increase in tumor size but no pathological fracture (Fig. 3). Because the diagnosis was OFD, her pain was first treated with painkillers but was not sufficiently controlled. We therefore decided to surgically remove the tibial tumor after thorough discussion with

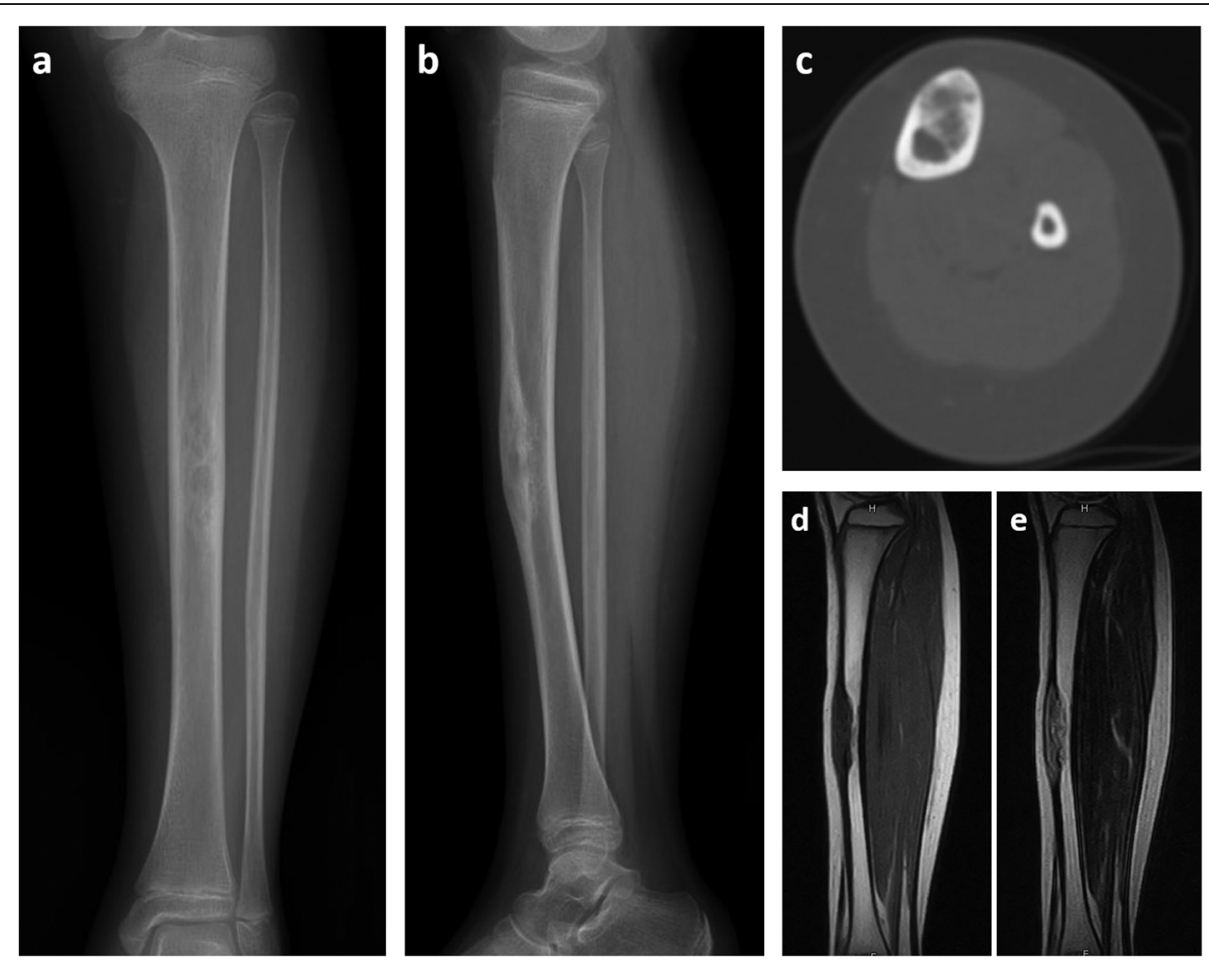

Fig. 1 The X-ray, CT image, and MR images of the tumor. a Anteroposterior radiograph. b Lateral radiograph. c Axial CT image. d T1-weighted magnetic resonance sagittal image. e T2-weighted magnetic resonance sagittal image 


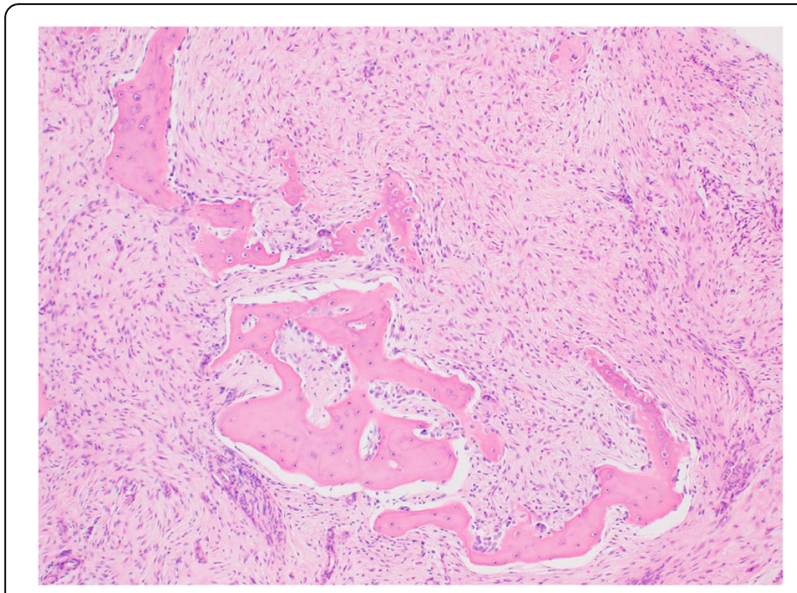

Fig. 2 The patient was diagnosed with osteofibrous dysplasia because there was no epithelial component in the biopsy specimen

her parents. Nine months after OFD diagnosis, intercalary $10-\mathrm{cm}$ segmental resection with $1.5-\mathrm{cm}$ wide partial cortex posteromedial preservation using a PVFG and a locking plate for reconstruction was performed. Resection was preceded by osteosynthesis using a locking plate to mechanically stabilize the lower leg. A $14-\mathrm{cm}$ section was removed from the patient's fibula; after reducing its size to $12 \mathrm{~cm}$, it was inlaid into medullary cavity of the tibia (Fig. 4a).
The tumor was composed of spindle cells with bony trabeculae rimmed by osteoblasts (Fig. 5a). Small clusters of epithelial cells were sparsely distributed throughout the tumor and were positive for AE1/AE3 immunostaining (Fig. 5b). Therefore, the diagnosis was upgraded to OFD-like AD. Bone union was observed 9 weeks after surgery (Fig. 4b), at which time the patient was allowed to partially bear weight. Full weight-bearing was allowed 12 weeks postoperatively. No complications were observed. Eight months after surgery (Fig. 4c), the patient walks without a cane. Nineteen months after surgery, the plate was removed (Fig. 4d). Twenty-two months after the definitive surgery, no recurrence was observed.

\section{Discussion}

This case is of interest to clinicians for two reasons. First, it shows progression of OFD to OFD-like AD. Second, it describes the successful treatment of OFD-like $A D$ via segmental resection with partial cortex preservation using a PVFG and a locking plate for reconstruction. This surgical method may achieve earlier bone union than do other methods and be feasible for cases of OFD and OFD-like AD in which the cortex can be partially preserved.

In our case, the diagnosis was changed from OFD to OFD-like AD after examination of a surgical specimen.
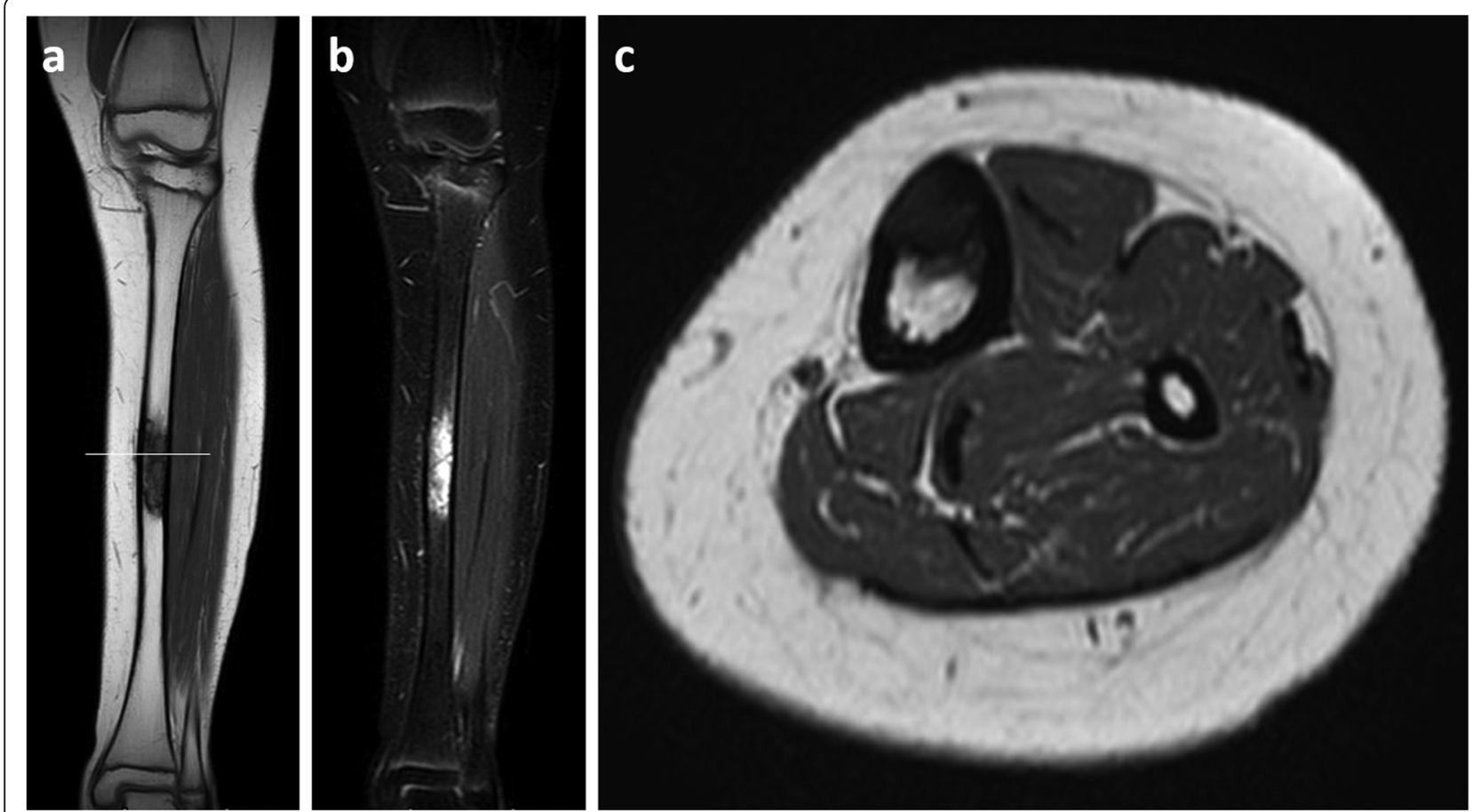

Fig. 3 The mass was a heterogeneously hypointense and isointense on a T1-weighted magnetic resonance image and $\mathbf{b}$ heterogeneously hyperintense on short TI inversion recovery magnetic resonance image. No pathological fracture was confirmed 


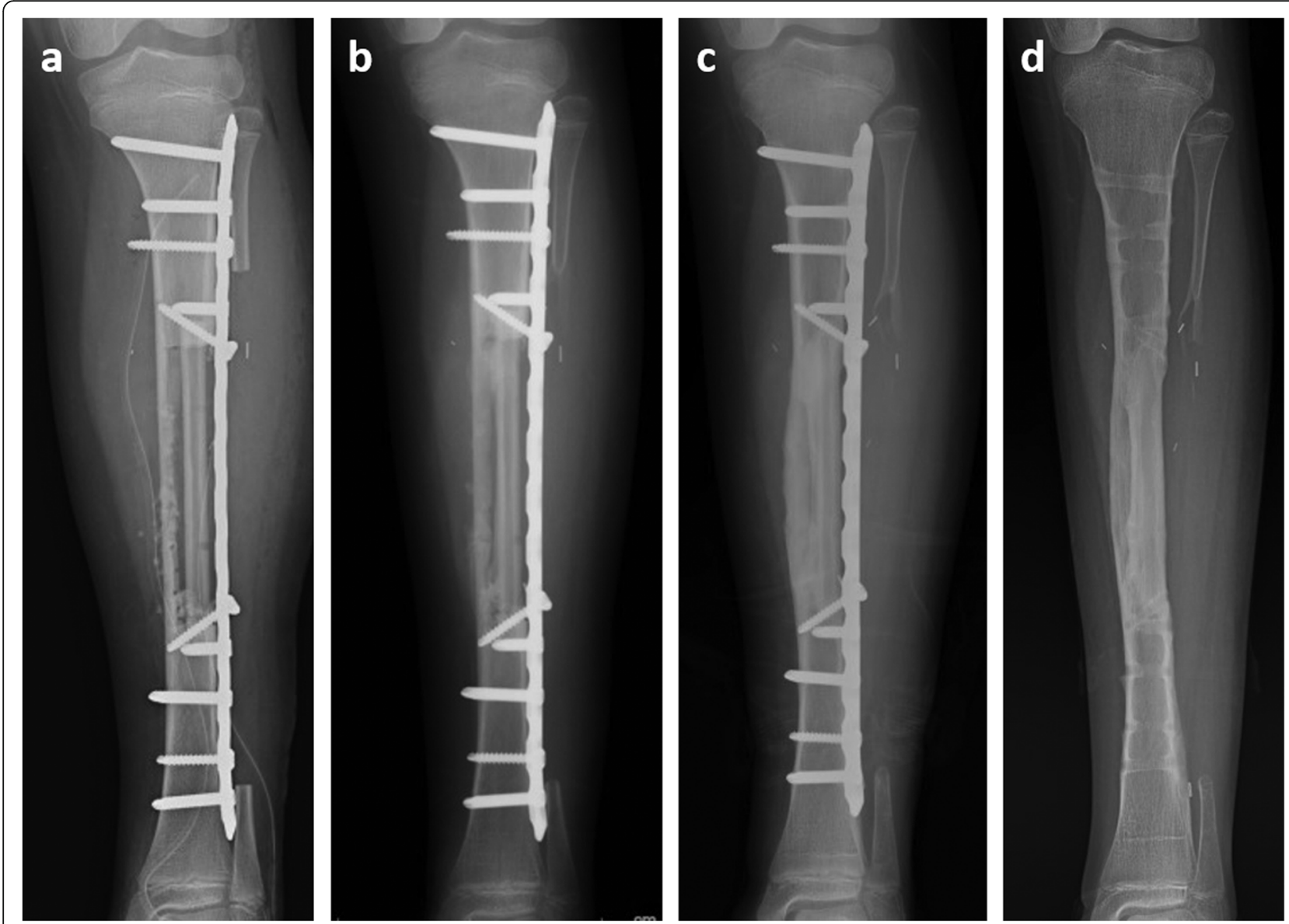

Fig. 4 a A locking plate was used to mechanically align and stabilize the lower leg. A pedicled vascularized fibula graft was inserted into the medullary cavity of the tibia and locked in place with cortical screws. b Bone union was observed 9 weeks after surgery, and the patient began to partially bear weight. c Complete bone union was observed 8 months after surgery. $\mathbf{d}$ The locking plate and screws were removed 19 months after surgery

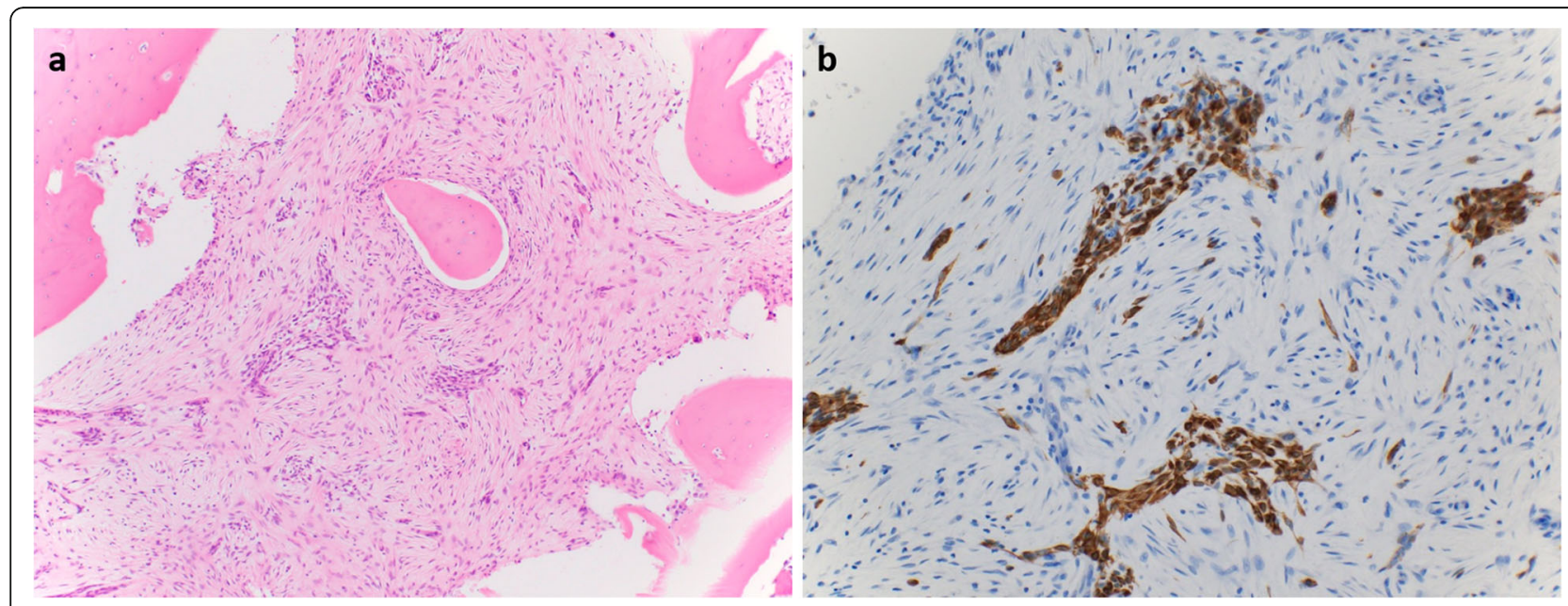

Fig. 5 The final diagnosis was osteofibrous dysplasia-like adamantinoma because the surgical specimen contained an epithelial component (a) with positive AE1/AE3 immunostaining (b) 
Progressive disease requiring surgery in patients with OFD-like AD initially treated for OFD has been reported $[6,7]$. Therefore, open biopsy is preferred to needle biopsy as it may better detect the small sparse clusters of epithelial cells that differentiate OFD-like AD from OFD. We performed open biopsy to prevent sampling errors, and the initial diagnosis was OFD [7]. The upgrade from OFD to OFD-like AD may represent a sampling error, but it is likely that disease progression had occurred. Patients with OFD may present with localized pain $(25-60 \%)[9,10]$. About $80 \%$ of OFD-like AD cases are accompanied by pain [6], and it is possible that tumors diagnosed as OFDs are actually OFD-like ADs in cases with severe pain.

Although our patient was initially diagnosed with OFD, we performed segmental resection because we could not control her pain. Several articles in recent years recommend radical segmental resection of OFDs or OFD-like ADs to prevent local recurrence $[6,7,11]$. The most popular reconstructive options after segmental resection of a bone tumor include intercalary allografts, VFGs, combined allografts and VFGs, endoprostheses, extracorporeal devitalized (i.e., irradiated, frozen, or pasteurized) autografts, and segmental transport based on the principles of distraction osteogenesis.

Intercalary allografts, the most widely accepted option, have a high incidence of nonunion, fracture, and infection. The use of a single devitalized autograft is also associated with nonunion. Therefore, for intercalary reconstruction, we prefer VFGs, which had good functional and oncological outcomes in the previous work of Emori et al. [8]. In VGFmediated reconstruction, bone union in the leg usually takes 5-7 months, but complications such as pseudarthrosis and fracture may arise. Hence, a strong fixation device such as a locking plate should be used to prevent these complications $[8,12]$. In the present case, segmental resection with partial cortex preservation using a PVFG and a locking plate expedited bone union (within 9 weeks after surgery) without complications, presumably because the partially preserved cortex better stabilized the affected limb.

\section{Conclusions}

We report a case of OFD-like AD that progressed from OFD and was treated via segmental resection with partial cortex preservation using a PVFG and a locking plate for reconstruction. This surgical method may provide early bone union and be feasible for cases of OFD and OFD-like AD in which the cortex can be partially preserved because of incomplete cortex involvement.

\section{Abbreviations}

OFD: Osteofibrous dysplasia; AD: Adamantinoma; VFG: Vascularized fibula graft

\section{Acknowledgements}

Not applicable

\section{Authors' contributions}

All authors designed the study and drafted the manuscript. All authors read and approved the final manuscript.

\section{Funding}

This work was partly supported by grants from JSPS KAKENHI (18K09112 to M. Emori).

\section{Availability of data and materials}

All data obtained is available within the manuscript.

\section{Ethics approval and consent to participate}

Written informed consent was obtained from the parents prior to publication of this case report, and all procedures performed were approved by the Institutional Review Board for Clinical Research at our university.

\section{Consent for publication}

Written informed consent was obtained from the parents to publish the information, including their photographs. A copy of the written consent is available for review.

\section{Competing interests}

The authors declare that they have no conflict of interest.

\section{Author details}

${ }^{1}$ Department of Orthopedic Surgery, Sapporo Medical University School of Medicine, West 16, South 1, Chuo- ku, Sapporo, Hokkaido 060-8543, Japan. ${ }^{2}$ Department of Diagnostic Pathology, Sapporo Medical University School of Medicine, Sapporo, Hokkaido, Japan.

Received: 20 May 2020 Accepted: 31 July 2020

Published online: 13 August 2020

\section{References}

1. WHO Classification of Tumours Editorial Board. WHO classification of tumours of soft tissue and bone 2020, Fifth ed.

2. Czerniak B, Rojas-Corona RR, Dorfman HD. Morphologic diversity of long bone adamantinoma. The concept of differentiated (regressing) adamantinoma and its relationship to osteofibrous dysplasia. Cancer. 1989; 64:2319-34

3. Most MJ, Sim FH, Inwards CY. Osteofibrous dysplasia and adamantinoma. J Am Acad Orthop Surg. 2010;18:358-66.

4. Gleason BC, Liegl-Atzwanger B, Kozakewich HP, Connolly S, Gebhardt MC, Fletcher JA, Perez-Atayde AR. Osteofibrous dysplasia and adamantinoma in children and adolescents: a clinicopathologic reappraisal. Am J Surg Pathol. 2008;32:363-76.

5. Ramanoudjame M, Guinebretiere JM, Mascard E, Seringe R, Dimeglio A, Wicart P. Is there a link between osteofibrous dysplasia and adamantinoma? Orthop Traumatol Surg Res. 2011;97:877-80.

6. Scholfield DW, Sadozai Z, Ghali C, Sumathi V, Douis H, Gaston L, Grimer RJ Jeys L: Does osteofibrous dysplasia progress to adamantinoma and how should they be treated? Bone Joint J 2017, 99-b:409-416.

7. Khanna M, Delaney D, Tirabosco R, Saifuddin A. Osteofibrous dysplasia, osteofibrous dysplasia-like adamantinoma and adamantinoma: correlation of radiological imaging features with surgical histology and assessment of the use of radiology in contributing to needle biopsy diagnosis. Skelet Radiol. 2008;37:1077-84.

8. Emori M, Kaya M, Irifune H, Takahashi N, Shimizu J, Mizushima E, Murahashi Y, Yamashita T: Vascularised fibular grafts for reconstruction of extremity bone defects after resection of bone and soft-tissue tumours: a single institutional study of 49 patients. Bone Joint J 2017, 99-b:1237-1243.

9. Park YK, Unni KK, McLeod RA, Pritchard DJ. Osteofibrous dysplasia: clinicopathologic study of 80 cases. Hum Pathol. 1993;24:1339-47.

10. Sweet DE, Vinh TN, Devaney K. Cortical osteofibrous dysplasia of long bone and its relationship to adamantinoma. A clinicopathologic study of 30 cases. Am J Surg Pathol. 1992;16:282-90. 
11. Lee RS, Weitzel S, Eastwood DM, Monsell F, Pringle J, Cannon SR, Briggs TW. Osteofibrous dysplasia of the tibia. Is there a need for a radical surgical approach? J Bone Joint Surg (Br). 2006;88:658-64.

12. Hilven PH, Bayliss L, Cosker T, Dijkstra PD, Jutte PC, Lahoda LU, Schaap GR, Bramer JA, van Drunen GK, Strackee SD, et al: The vascularised fibular graft for limb salvage after bone tumour surgery: a multicentre study. Bone Joint J 2015, 97-b:853-861.

\section{Publisher's Note}

Springer Nature remains neutral with regard to jurisdictional claims in published maps and institutional affiliations.

Ready to submit your research? Choose BMC and benefit from:

- fast, convenient online submission

- thorough peer review by experienced researchers in your field

- rapid publication on acceptance

- support for research data, including large and complex data types

- gold Open Access which fosters wider collaboration and increased citations

- maximum visibility for your research: over $100 \mathrm{M}$ website views per year

At $B M C$, research is always in progress.

Learn more biomedcentral.com/submissions 